\title{
La création ne serait-elle pas le contraire de la communication?
}

Wouldn't creation mean the opposite of widespread circulation?

\section{André De Baecque}

\section{CpenEdition}

Journals

Édition électronique

URL : http://journals.openedition.org/edc/2797

DOI : $10.4000 /$ edc. 2797

ISSN : 2101-0366

Éditeur

Université Lille-3

Édition imprimée

Date de publication : 1 mai 1991

Pagination : 117-126

ISSN : $1270-6841$

Référence électronique

André De Baecque, "La création ne serait-elle pas le contraire de la communication ? ", Études de communication [En ligne], 12 | 1991, mis en ligne le 19 janvier 2012, consulté le 20 avril 2019. URL: http://journals.openedition.org/edc/2797 ; DOI : 10.4000/edc.2797

Ce document a été généré automatiquement le 20 avril 2019

(c) Tous droits réservés 


\title{
La création ne serait-elle pas le contraire de la communication?
}

Wouldn't creation mean the opposite of widespread circulation?

\author{
André De Baecque
}

1 Je suis un bouffon triste et sans grâce qui prétend " faire le sot métier d'auteur dramatique ", au moment où l'opinion la plus couramment véhiculée, notamment par les médias audiovisuels, prétend qu'il n'y a plus d'écrivains de théâtre en France.

2 Aussi comprendra-t-on que je sois tout à la fois étonné, intimidé et flatté d'être partie prenante dans un aéropage d'universitaires, de chercheurs, de penseurs, de " sachants » qui s'interrogent entre autres sur la communication culturelle, vaste sujet que je souhaite, en ce qui me concerne, limiter au domaine où je peux faire état d'une pratique et d'une expérience concrètes, c'est-à-dire le spectacle vivant.

3 C'est alors l'occasion de poser par paliers des questions aussi naïves qu'existentielles: Qu'est-ce que la création (notamment théâtrale) ? Quelles sont les chances de survie du théâtre face à la prolifération des industries culturelles ? Quelles sont les caractéristiques de l'information culturelle?

\section{Qu'est-ce que la création?}

4 A une époque où le mot fait florès, où l'on qualifie de " créatif » tout ce que la mode pousse sur le devant de la scène médiatique, les concepteurs publicitaires, les stylistes, les interprètes, les banquiers, les cyclistes, les maîtres queue, les animateurs audiovisuels de tous calibres et de toutes ambitions, il n'est pas inutile de revenir à certaines définitions, certains critères, sans lesquels toute tentative d'analyse est un leurre.

5 La création est donc d'abord et avant tout un prototype. C'est-à-dire une oeuvre complètement originale et inédite. C'est le fait d'un individu isolé - même si une équipe nombreuse et diverse travaille pour lui -, qui a l'utopie ou l'outrecuidance de prétendre intéresser d'autres personnes à son univers. 
6 Lorsqu'il arrive à percer le mur de l'indifférence, ce prototype, par sa novation, est généralement perçu comme un objet de subversion et de scandale.

7 On oublie trop souvent que Molière, menacé de mort, a dû renoncer à jouer Don Juan ou que des rixes mortelles éclataient sur les gradins de l'amphithéâtre lorsque les spectateurs découvraient pour la première fois une tragédie de Sophocle.

Ce qui est évident également, c'est que ce prototype a été conçu sans aucun souci de rentabilité. Rappelons pour mémoire que Marcel Proust a publié à compte d'auteur les premiers tomes d'A la recherche du temps perdu, que Jean Cocteau a projeté Le sang d'un poète devant une poignée de fidèles, quant à Van Gogh ou à Modigliani...

9 - La création est le contraire d'un phénomène médiatique qui est oublié au bout de quelques jours ou de quelques semaines et remplacé par un phénomène de même nature. La création/prototype est un jalon dans l'oeuvre d'un artiste. Les Quatuors de Beethoven renvoient à ses symphonies qui ont des correspondances avec ses concertos, ses sonates, ses bagatelles. De même Nostalghia de Tarkovski s'inscrit dans la lignée de L'Enfance d'Ivan, de Solaris, du Miroir, de Stalker. De même également les romans de Jean Genêt, Notre Dame des fleurs, Le miracle de la rose, Pompes funèbres, annoncent les oeuvres dramatiques majeures, Les bonnes, Le balcon, Les nègres, Les paravents. De même toujours les chorégraphies de Jean Claude Gallotta pour le Groupe Émile Dubois se renvoient l'une à l'autre dans un labyrinthe de gestes et d'actions où le besoin de se toucher, de s'aimer, de se battre, d'exister, est symbolisé par le titre d'une des pièces les plus étonnantes du groupe, Les survivants.

10 A contrario, on peut se demander, au cas où l'on ne connaîtrait de Molière que La jalousie du barbouillé ou Le cocu imaginaire, si on le considèrerait comme un génie de l'écriture dramatique.

11 - La création est par ailleurs une ascèse artisanale d'où se dégage la forme d'un style, d'une écriture. Ainsi la façon de composer de Stockhausen, la façon de filmer de Jean Luc Godard, la façon d'écrire de Claude Simon ou de Samuel Beckett sont autant de repères, de signes de reconnaissance.

12 - La création enfin, tout en prenant en compte la sensibilité d'une époque, s'inscrit dans la durée. Ayant résisté à l'épreuve du temps, même si à ses débuts elle a été contestée ou ignorée, elle obtient le statut de classique et devient une référence pour les générations à venir.

13 - A l'inverse de la création, le produit culturel fabriqué par les industries du même nom, doit avoir le contenu le plus efficace, le plus immédiatement perceptible et le plus international possible.

14 C'est donc une marchandise dont la production a réclamé des investissements souvent considérables et dont la promotion doit être à la hauteur des risques financiers encourus. Le lancement de ce produit deviendra un événement aussi spectaculaire qu'éphémère. Et c'est pour pallier les inconvénients d'un oubli trop rapide et accéléré par la concurrence que les investisseurs cherchent à démultiplier les applications de ce produit en lui faisant parcourir la chaîne des différentes techniques d'expression, film, émission tv, livre, bd, gadget, reproduction grandeur nature des héros popularisés par l'image dans les Parcs de Loisirs... 


\section{Le théâtre est-il encore un lieu de création?}

15 A partir des critères énoncés ci-dessus, on peut se demander si le théâtre est encore un lieu de création.

16 Cette interrogation justifiée (le Directeur du Théâtre au Ministère de la Culture, Monsieur Faivre d'Arcier, n'a-t-il pas annoncé récemment que le théâtre avait pratiquement disparu dans des pays aussi développés que les États-Unis et le Japon, cf. Télérama 20.12.89) oblige bien entendu à nuancer et à préciser les choses. Car le théâtre avec un grand $\mathrm{T}$ n'existe pas. Ce n'est pas une entité abstraite ; c'est une pratique indéfiniment renouvelée et démultipliée. Il y a chaque soir en France et dans le monde des milliers de représentations et de publics divers. On peut jouer une pièce des années entières. Chaque représentation sera différente de la précédente, selon la plus ou moins grande réceptivité des publics qui se succèdent et qui ont une influence directe sur l'interprétation des comédiens.

17 Rappelons que le théâtre est également et tout à la fois un parcours, une fête et une pratique et que les milliers de comédiens amateurs qui consacrent avec une passion et un désintéressement exemplaires leurs loisirs à l'art dramatique constituent le terreau à partir duquel le théâtre professionnel peut s'épanouir et progresser.

18 Cessons donc d'appréhender le théâtre en termes quantitatifs et statistiques propres aux mass-médias. Le théâtre doit revendiquer la fierté d'être un « art élitaire », en bannissant de cette qualification toute connotation financière ou mandarinale. Cette fonction élitaire repose essentiellement sur le volontariat, la démarche active. On ne subit pas des images et des sons. On choisit d'écouter un texte et de participer à un jeu.

19 Car finalement, et contrairement à la plupart des autres pratiques culturelles, le théâtre constitue l'art de la relation directe entre un auteur qui propose une histoire, des comédiens qui l'interprètent, des publics qui l'acceptent ou qui la rejettent. C'est en ce sens que l'on peut affirmer sans crainte d'être démenti que la pratique théâtrale est assurée de sa pérennité.

20 Tant que les hommes vivront en société, ils éprouveront le besoin de s'assembler pour vérifier qu'ils ont en commun des sentiments, une sensibilité et l'envie de se distraire. Ce besoin communautaire deviendra même l'antidote indispensable à la soliture engendrée par la sophistication de plus en plus poussée de nos technologies de communication qui nous transforment en monades.

\section{Parler d'aujourd'hui.}

21 Relation directe veut dire parler d'aujourd'hui, comme le font du reste les chorégraphes et les réalisateurs de films. Or le théâtre dans notre pays est menacé et perverti par deux maladies : le poids des Institutions et la fascination des Médias audio-visuels.

Pendant vingt cinq ans - en gros de la Libération au début des années 1970 -, la décentralisation dramatique a été un projet, puis un début de réalité vivants, dynamiques, mobilisateurs. Des Institutions culturelles ont été créées avec des statuts et des hiérarchies dont les échelons évoquent le parcours du combattant. Ces Institutions se sont peu à peu fonctionnarisées, complexifiées, et dans bien des cas ankylosées et pétrifiées. 

des prototypes d'écriture dont l'ambition, l'envergure, l'originalité présentent des équivalences avec Les Atrides, Troilus et Cressida, Don Juan ou Le soulier de satin (" La scène du théâtre est le monde... ». Quelle invite à s'envoler !). L'époque pourrait s'y prêter, semblet-il.

\section{déclare (cf. Art Press, Février 1989) :}

Si les auteurs veulent vraiment être joués, qu'ils se mettent en scène eux-mêmes, au lieu de se comporter comme d'éternels assistés.

35

Études de communication, 12 | 1991 
36 Alors si on disait chiche à ces directeurs qui font la leçon aux auteurs, en leur tenant le langage suivant :

- Si vous montez Shakespeare, Molière ou Marivaux, vous avez déjà un public, l'aide de l'État est inutile. Par contre, si vous montez l'oeuvre inédite d'un auteur vivant...

Cela s'est fait en Grande-Bretagne au cours des années 1960. Des centaines d'auteurs nouveaux ont été révélés, mais pas un metteur en scène. Le seul qui est devenu une vedette internationale s'est installé en France. Il s'agit de Peter Brook.

\section{La fascination des industries culturelles.}

La fascination des médias audiovisuels, de l'image, et singulièrement de la télévision, est pour le théâtre un autre risque majeur, celui de tomber de Charybde en Scylla et de se laisser engloutir par des sirènes chatoyantes. A partir du moment où l'écriture dramatique est complétée, et dans certains cas occultée, par l'écriture scénique, l'esthétisme devient une finalité. Car l'écriture scénique privilégie l'image.

L'art dramatique se transforme en tableaux, animés par toutes les ressources de l'invention plastique et de la technologie. A ce stade, la concurrence entre metteurs en scène est redoutable, surtout lorsqu'elle s'exerce sur un même sujet. Il arrive par exemple que Don Juan du toujours jeune Molière soit monté cinq fois au cours de la même saison.

Dans cette compétition d'un genre particulier, la consécration récompensera celui qui aura su allier le prestige de la distribution, la sophistication de la machinerie, et « les trouvailles » de mise en scène.

41 On assiste alors à une inflation des coûts de production. Robert Abirached, faisant le bilan de " son septennat " à la Direction du Théâtre, constatait que les budgets de mise en scène avaient triplé en quelques années, pour atteindre dans certains cas des niveaux comparables à ceux de la production d'un film.

42 Nous passons alors de la catégorie création/prototype à celle de produit culturel, avec au moins trois conséquences particulièrement nocives :

- Un club très fermé dit « haut de gamme » se constitue, regroupant les institutions de prestige, seules susceptibles de financer des opérations placées sous le signe du vedettariat et de la technologie.

- Ce n'est plus le répertoire qui se renouvelle, mais la représentation que l'on s'en fait. Ce règne de l'apparence s'institue au détriment de la découverte authentique. Le contenu disparaît au bénéfice du contenant, des moyens mis en oeuvre, véritable finalité de l'opération.

- La promotion d'un tel système s'apparente aux pratiques du show business.

43 La notion de " coup médiatique ", outre qu'elle nous paraît contraire à la spécificité même du théâtre, amène des esprits faussement naïfs à se poser des questions pernicieuses du type : Pourquoi dépenser des sommes relativement considérables pour réunir dans un lieu de spectacle au maximum quelques milliers de personnes, alors que le même spectacle diffusé à la télévision sera vu par des millions de téléspectateurs? 


\section{La télévision et la notion de spectacle.}

d'écoute et d'attention des spectateurs de théâtre qui supportent plus difficilement un spectacle dont la durée dépasse une heure et demie (espace d'un film de long métrage ou d'une émission de télévision). (du propos, du lieu, de la durée, du nombre de personnages). Si ce n'est pas trop cher, je serai plus facilement monté, et peut-être que la déesse télévision me fera les yeux doux...

53 Cette frustration, cette auto censure ne facilitent pas l'éclosion, le jaillissement d'une création protéiforme. Quel serait le fou qui se risquerait aujourd'hui à passer une partie de sa vie sur l'équivalent du manuscrit de Peer Gynt ou du Soulier de satin? N'est-ce pas pourtant de cela que le théâtre vivant aurait le plus urgent besoin?

\section{Ne pas confondre communication et information culturelle.}

On l'aura deviné par les développements qui précèdent, j'éprouve une méfiance instinctive pour le mot " communication ", fourre tout sémantique où l'on peut pêcher, au gré de son humeur ou de ses analyses, des théories, des statistiques, des techniques, des réseaux, des procédés de marketing, l'existence d'un marché, et le besoin subjectif de « communier avec ».

La communication recoupe en effet l'espace économique des industries culturelles, c'està-dire les moyens (recherches, investissements, machines) qui concourent à la production 
et à la reproduction des images, des sons, des éditions, de tout ce qui relève du loisir, et par extension également les produits culturels diffusés.

En second lieu, le service communication d'une entreprise (culturelle ou non) regroupe les spécialistes de l'élaboration, de la promotion et de la diffusion d'un message qui utilisent les techniques de la publicité, des relations publiques et de l'information destinée à la presse.

Enfin accessoirement, la communication c'est le message lui-même.

En quoi ce faisceau de vecteurs qui confondent allègrement le contenant et le contenu peut-il concerner le théâtre?

En rien, serait-on spontanément tenté de répondre.

60 Art élitaire et de relation directe qui, à chaque représentation, rassemble au maximum un millier de spectateurs, le théâtre possède une originalité, une spécificité, qui en font exactement le contraire d'un produit issu des mass médias.

61 Par ailleurs la production théâtrale n'a ni la vocation ni les moyens d'être le prolongement ou l'ébauche d'une grille de programmes télévisés. Le problème n'est pas d'articuler le théâtre et l'image, mais de préserver des lieux où l'on peut se passer de la télévision.

Gilles Deleuze l'affirme : La création est le contraire de la communication. Georges Lavaudant le constate également dans un article intitulé, « Le Théâtre, ce n'est pas la communication » (cf. Le Monde, 2.10.1987) :

Après tant d'efforts pour banaliser l'acte d'aller au théâtre, il faut maintenant retrouver une force de déclic. Aujourd'hui bien souvent, on essaie de monter les spectacles les meilleurs, l'un après l'autre, sans se préoccuper de l'identité du théâtre.

Je crains la confusion entre les spectacles et le bruit fait autour, qui dénature, non pas le travail lui-même, mais le contact entre le public et la représentation, la rencontre.

63 Cette identité du théâtre, n'est-ce pas précisément à l'information culturelle d'en assumer la préoccupation ? Il y a tellement de clichés qui ont la vie dure parce qu'ils sont perpétués par les médias de grande écoute. Je cite : " Le théâtre est un moyen d'expression inefficace, obsolète et ennuyeux... ». " Il nourrit plus de parasites qu'il ne contente de spectateurs... ». Au reste, « les auteurs en renom n'écrivent plus de pièces... », etc...

64 Ce travail de réhabilitation qui consiste à montrer que le théâtre est un art divers, multiple, authentiquement convivial, et qui parle d'aujourd'hui, n'est-ce pas ce que l'on appelait autrefois l'animation culturelle et que l'on a peut-être un peu prématurément enterrée.

Car si les spectateurs d'une création théâtrale ne sont plus des partenaires, mais de simples clients que l'on tente d'allécher par les procédés de la promotion commerciale, alors oui, ce théâtre-là est très malade. Il n'a même plus de raisons valables d'exister. 


\section{RÉSUMÉS}

Pour A. De Baecque, auteur dramatique, la création vaut comme prototype, jalon d'une oeuvre, recherche d'une écriture propre à la recherche de pérennité. La représentation théâtrale est alors le moment d'une relation directe entre l'auteur, le comédien, le public ; le théâtre est du coup un « art élitaire ».

La « communication culturelle » que l'on voit se développer, valorisant le metteur en scène et insistant sur le spectaculaire, est plus proche des industries culturelles au risque de trahir l'essence du théâtre. L'auteur estime qu'une telle communication met en danger l'écriture dramatique et le renouvellement du répertoire. Il prône un retour à « l'information culturelle ».

For A.De Baecque, a playwright, each individual creation is a prototype, the significant mark of a work, the search for a perennial writing. Then the performance of a play is a moment of direct contact between the playwright, the actors and the public; consequently the theatre is an art for the " happy few ». The cultural advertisements which are being developed now give the chief role to the art director to the spectacular impact and as they are much nearer to the show industry they might betray the essential meaning of theatre. The writer thinks that such a cultural communication is a danger for dramatic writing and the progression towards new forms of creation. He highly recommends a return to a real cultural information.

INDEX

Mots-clés : création artistique, théâtre, public, communication culturelle, industrie culturelle, télévision, politique culturelle

Keywords : artistic creation, theatre, audience, cultural communication, cultural industry, television, cultural policy

\section{AUTEUR}

\section{ANDRÉ DE BAECQUE}

André de Baecque, auteur dramatique 